\title{
Convergence fields of regular matrix transformations of sequences of elements of Banach spaces
}

\author{
T. Visnyai
}




\title{
CONVERGENCE FIELDS OF REGULAR MATRIX TRANSFORMATIONS OF SEQUENCES OF ELEMENTS OF BANACH SPACES
}

\author{
T. VISNYAI
}

[Received: May 23, 2005]

\begin{abstract}
AвstRact. In $[4,7]$ some properties of convergence fields of regular matrix transformations of bounded sequences of real numbers are presented. We shall prove a generalization of Steinhaus' theorem for sequences of a Banach space and show that results of [4] cen be generalize for a space of sequences of elements of a Banach space $(X,\|\cdot\|)$.
\end{abstract}

Mathematics Subject Classification: 46B45,40D09

Keywords: convergence field, Baire category, porosity

\section{InTRODUCTION}

Let $(X,\|\cdot\|)$ be a Banach space. The sequence $\alpha=\left(\alpha_{k}\right)$ converges to $c$ if $\forall \varepsilon>0$ $\exists k_{0} \in \mathbb{N} \forall k>k_{0}:\left\|\alpha_{k}-c\right\|<\varepsilon$. We write $\lim _{k \rightarrow \infty} \alpha_{k}=c$.

We define the following sets:

(a) $b=\left\{\alpha=\left(\alpha_{k}\right): \alpha_{k} \in X, k=1,2, \ldots\right\}$;

(b) $B_{\infty}=\left\{\alpha=\left(\alpha_{k}\right): \alpha_{k} \in X, k=1,2, \ldots: \exists K_{\alpha}>0 \forall k=1,2, \ldots\left\|\alpha_{k}\right\| \leq K_{\alpha}\right\}$;

(c) $\Omega=\left\{\alpha=\left(\alpha_{k}\right): \alpha_{k} \in X, k=1,2, \ldots: \forall k=1,2, \ldots\left\|\alpha_{k}\right\|=0 \vee\left\|\alpha_{k}\right\|=1\right\}$.

The set $b$ contains all sequences of elements of $X$, the set $B_{\infty}$ is the set of all bounded sequences of $X$ and $\Omega$ is the set of all sequences of elements of $X$ which have norm null or one. In what follows, for the set $B$, the following inclusions hold:

$$
\Omega \subset B_{\infty} \subset B \subset b .
$$

The notion of porosity has been introduced in [10]. It is a suitable tool to describe small sets in a metric space.

Let $(Y, d)$ be a metric space, $Z \subset Y$. Let $y \in Y, \delta>0$, and let $B(y, \delta)$ denote the set $\{x \in Y: d(x, y)<\delta\}$. We put

$P(y, Z, \delta)=\sup \{t>0: \exists z \in B(y, \delta)$ such that $B(z, t) \subset B(y, \delta)$ and $B(z, t) \cap Z=\varnothing\}$.

Supported by the Grant VEGA 1/3018/06. 
If such $t>0$ does not exist, we put $P(y, Z, \delta)=0$. The numbers

$$
\underline{p}(y, Z)=\liminf _{\delta \rightarrow 0^{+}} \frac{P(y, Z, \delta)}{\delta}
$$

and

$$
\bar{p}(y, Z)=\limsup _{\delta \rightarrow 0^{+}} \frac{P(y, Z, \delta)}{\delta}
$$

are called the lower and upper porosity of the set $Z$ at $y \in Y$, respectively.

A set $Z \subset Y$ for which $\bar{p}(y, Z)>0$ for every $y \in Y$ is said to be porous in $Y$. Obviously every set porous in $Y$ is nowhere dense in $Y$. If $p(y, Z)=\bar{p}(y, Z)=p(y, Z)$, then the number $p(y, Z)$ is called porosity of $Z$ at $y \in \bar{Y}$. If $p(y, Z)=1$, then $Z$ is said to be strongly porous at $y$. If for all $y \in Y$ we have $p(y, Z)=1$ for $y \notin Z$ and $p(y, Z)=\frac{1}{2}$ for $y \in Z$, then $Z$ is said to be strongly porous at $Y$. The set $W$ is said to be $\sigma$-porous ( $\sigma$-strongly porous) at $Y$ if $W=\bigcup_{n=1}^{\infty} Z_{n}$ and each $Z_{n}$ is porous (strongly porous) at $Y$.

Further on, we recall the definition of a matrix transformation. Let $\mathscr{A}=\left(a_{n k}\right)$ be an infinite matrix of real numbers. A sequence $\alpha=\left(\alpha_{k}\right), \alpha_{k} \in X$, is said to be $\mathscr{A}$-limitable (limitable by the method $\mathscr{A}$ ) to the element $c \in X$ if, for $\beta=\left(\beta_{n}(\alpha)\right.$ ), $\beta_{n}=\beta_{n}(\alpha)=\sum_{k=1}^{\infty} a_{n k} \alpha_{k}$, we have $\lim _{n \rightarrow \infty} \beta_{n}=c$.

If $\alpha=\left(\alpha_{k}\right)$ is $\mathscr{A}$-limitable to the element $c$, we write $\mathscr{A}-\lim _{k \rightarrow \infty} \alpha_{k}=c$. The method $\mathscr{A}$ defined by the matrix $\mathscr{A}$ is said to be regular if $\lim _{k \rightarrow \infty} \alpha_{k}=c$ implies that $\mathscr{A}-\lim _{k \rightarrow \infty} \alpha_{k}=c$.

Let $\mathscr{A}$ be a regular method. The symbol $F(\mathscr{A})$ denotes the set of all $\mathscr{A}$-limitable sequences of $X$. We put

$$
\begin{aligned}
F(\mathscr{A})=\left\{\alpha=\left(\alpha_{k}\right) \mid \alpha_{k} \in X, k=1,2, \ldots: \text { there exists } \lim _{n \rightarrow \infty} \beta_{n},\right. \\
\text { where } \left.\beta_{n}=\sum_{k=1}^{\infty} a_{n k} \alpha_{k}\right\} .
\end{aligned}
$$

The set $F(\mathscr{A})$ is called the convergence field of the matrix transformation $\mathscr{A}$.

It is proved in [4] that $F(\mathscr{A})$ is a set of first Baire category in $S$, where $S$ is a linear space of sequences of real numbers and $F(\mathscr{A})$ is strongly porous in $l_{\infty}\left(l_{\infty}\right.$ is the set of all bounded sequences of real numbers).

In this paper we show that these results can be generalized for the sequences of elements of Banach space $(X,\|\cdot\|)$.

\section{Main Results}

Monograph [6] gives the Toeplitz theorem which provides necessary and sufficient condition for matrix $\mathscr{A}$ be a regular, i. e., when $\lim _{k \rightarrow \infty} x_{k}=t$ implies $\mathscr{A}-\lim _{k \rightarrow \infty} x_{k}=$ $t$ for real sequences $\left(x_{k}\right)$. 
In [9], it is proved that Toeplitz theorem holds also for sequences of elements of a Banach space.

Theorem A. Let $(X,\|\cdot\|)$ be a Banach space. Let $\mathscr{A}=\left(a_{n k}\right)$ be an infinite matrix of real numbers. The necessary and sufficient condition for a sequence $\beta_{n}(\alpha)=$ $\sum_{k=1}^{\infty} a_{n k} \alpha_{k}$, to converge to $c \in X$ as $n \rightarrow \infty$ where $\lim _{k \rightarrow \infty} \alpha_{k}=c, \alpha_{k} \in X$ is that matrix $\mathscr{A}$ satisfies the following three conditions:

(a) $\exists M>0, \forall n=1,2, \ldots \sum_{k=1}^{\infty}\left|a_{n k}\right| \leq M$;

(b) $\forall k=1,2, \ldots \lim _{n \rightarrow \infty} a_{n k}=0$;

(c) $\lim _{n \rightarrow \infty} \sum_{k=1}^{\infty} a_{n k}=1$.

Further we will use the following theorem concerning the functions of Baire class one (see [8, p. 185]).

Theorem B. Let $A$ and $B$ be metric spaces. Let $\delta_{n}: A \rightarrow B, n=1,2, \ldots$, be a sequence of continuous operators, which converges pointwise to an operator $\delta$, i. e.,

$$
\forall a \in A: \lim _{n \rightarrow \infty} \delta_{n}(a)=\delta(a)
$$

Then the set of all discontinuity points of the operator $\delta: A \rightarrow B$ is a set of the first Baire category.

In [2], the Steinhaus theorem is proved under the condition that there does not exist a regular matrix which limits all sequences of 0's and 1's. Now we will show an analogue of the Steinhaus theorem for sequences of elements of $X$. First we prove the following:

Lemma 1. The metric space $(\Omega, d)$, where $d(\alpha, \beta)=\sum_{k=1}^{\infty} 2^{-k}\left\|\alpha_{k}-\beta_{k}\right\|, \alpha=$ $\left(\alpha_{k}\right) \in \Omega, \beta=\left(\beta_{k}\right) \in \Omega$, is a complete metric space.

Proof. The function $d: \Omega \times \Omega \rightarrow\langle 0, \infty)$ is a metric.

Let $\alpha^{(n)}=\left(\alpha_{k}^{(n)}\right), n=1,2, \ldots$ be a Cauchy sequence of elements of $\Omega$. Thus,

$$
\forall \varepsilon>0 \exists n_{0} \in \mathbb{N} \quad \forall m, n>n_{0}: d\left(\alpha^{(n)}, \alpha^{(m)}\right)<\varepsilon .
$$

Choose $j \in \mathbb{N}$. Then

$$
\varepsilon>d\left(\alpha^{(n)}, \alpha^{(m)}\right)=\sum_{k=1}^{\infty} \frac{\left\|\alpha_{k}^{(n)}-\alpha_{k}^{(m)}\right\|}{2^{k}} \geq \frac{1}{2^{j}}\left\|\alpha_{j}^{(n)}-\alpha_{j}^{(m)}\right\| .
$$

The sequences $\left(\alpha_{j}^{(n)}\right)_{n=1}^{\infty}$ of elements of $X$ are Cauchy sequences. $X$ is a complete metric space, therefore $\left(\alpha_{j}^{(n)}\right)_{n=1}^{\infty}$ is convergent.

Let $\lim _{n \rightarrow \infty} \alpha_{j}^{(n)}=\alpha_{j}$. If $\alpha=\left(\alpha_{j}\right)_{j=1}^{\infty}$, then one can easily verify that that $\lim _{n \rightarrow \infty} \alpha^{(n)}=$ $\alpha$ and, for each $j=1,2, \ldots,\left\|\alpha_{j}\right\|=0$ or $\left\|\alpha_{j}\right\|=1$.

Theorem C. For any regular matrix $\mathscr{A}=\left(a_{n k}\right)$ there exists a sequence in the set $\Omega$, which is not limitable by the method $\mathscr{A}$. 
Proof. Let $\mathscr{A}=\left(a_{n k}\right)$ be a regular matrix. Then there exists an $M>0$ such that, for all $n=1,2, \ldots$,

$$
\sum_{k=1}^{\infty}\left|a_{n k}\right| \leq M
$$

We prove that the operator $\delta_{n}: \Omega \rightarrow X$ is continuous at $\alpha=\left(\alpha_{k}\right) \in \Omega$ for each $n=1,2, \ldots$, where $\delta_{n}(\alpha)=\sum_{k=1}^{\infty} a_{n k} \alpha_{k}, \alpha=\left(\alpha_{k}\right) \in \Omega$. Let $\varepsilon>0$ and $\alpha=\left(\alpha_{k}\right) \in$ $\Omega, \beta=\left(\beta_{k}\right) \in \Omega$. Then

$$
\left\|\delta_{n}(\alpha)-\delta_{n}(\beta)\right\|=\left\|\sum_{k=1}^{\infty} a_{n k} \alpha_{k}-\sum_{k=1}^{\infty} a_{n k} \beta_{k}\right\| \leq \sum_{k=1}^{\infty}\left|a_{n k}\left\|\mid \alpha_{k}-\beta_{k}\right\| .\right.
$$

We choose $n_{0} \in \mathbb{N}$ so that $\sum_{k>n_{0}}\left|a_{n k}\right|<\frac{\varepsilon}{4}$. Then

$$
\sum_{k>n_{0}}\left|a_{n k}\right|\left\|\alpha_{k}-\beta_{k}\right\| \leq \sum_{k>n_{0}}\left|a_{n k}\right|\left(\left\|\alpha_{k}\right\|+\left\|\beta_{k}\right\|\right) \leq 2 \cdot \frac{\varepsilon}{4}=\frac{\varepsilon}{2} .
$$

If $d(\alpha, \beta)<\frac{\varepsilon}{2 M 2^{n} 0}$ for $\alpha$ and $\beta$, then we have

$$
\frac{\left\|\alpha_{k}-\beta_{k}\right\|}{2^{k}} \leq d(\alpha, \beta)<\frac{\varepsilon}{2 M 2^{n_{0}}}
$$

for all $k=1,2, \ldots n_{0}$. Therefore, $\left\|\alpha_{k}-\beta_{k}\right\|<\frac{\varepsilon}{2 M}$ and

$$
\sum_{k=1}^{n_{0}}\left|a_{n k}\right|\left|\alpha_{k}-\beta_{k}\right|\left|<\frac{\varepsilon}{2 M} \sum_{k=1}^{n_{0}}\right| a_{n k} \mid \leq \frac{\varepsilon}{2} .
$$

According to (2)-(4), the inequality $d(\alpha, \beta)<\frac{\varepsilon}{2 M 2^{n_{0}}}$ implies that $\left\|\delta_{n}(\alpha)-\delta_{n}(\beta)\right\|<\varepsilon$. The operator $\delta_{n}, n=1,2, \ldots$, is continuous at $\alpha=\left(\alpha_{k}\right)$.

Suppose that all sequences in $\Omega$ are limitable by the matrix $\mathscr{A}=\left(a_{n k}\right)$. Hence, there exists $\lim _{n \rightarrow \infty} \delta_{n}(\alpha)=\delta(\alpha)$ for each $\alpha=\left(\alpha_{k}\right) \in \Omega$. Then, by Theorem B, the set of discontinuity points of the operator $\delta: \Omega \rightarrow X$ is a set of the first Baire category in $\Omega$. On the other hand if $\alpha=\left(\alpha_{k}\right) \in \Omega$ and $\eta>0$, then, in the open ball $S(\alpha, \eta)$, it is possible to find elements $\beta, \gamma \in \Omega$ such that

We choose $k_{1} \in \mathbb{N}$ so that

$$
\|\delta(\beta)-\delta(\gamma)\|>\frac{1}{2} .
$$

$$
\sum_{k>k_{1}} \frac{\left\|\alpha_{k}\right\|}{2^{k}} \leq \sum_{k>k_{1}} \frac{1}{2^{k}}<\frac{\eta}{4},
$$

where, $\alpha=\left(\alpha_{k}\right) \in \Omega$. Put $\beta=\left(\beta_{k}\right), \gamma=\left(\gamma_{k}\right)$, where $\beta_{k}=\gamma_{k}=\alpha_{k}$ for $k=1,2, \ldots k_{1}$ and $\beta_{k}=\Theta, \gamma_{k}=\xi$ for $k>k_{1}$. The element $\Theta$ is the null-element of $X$ and $\xi \in X$ is an element with the property $\|\xi\|=1$. Then

$$
d(\alpha, \beta)=\sum_{k>k_{1}} \frac{\left\|\alpha_{k}-\Theta\right\|}{2^{k}}<\frac{\eta}{4}
$$


and

$$
d(\alpha, \gamma)=\sum_{k>k_{1}} \frac{\left\|\alpha_{k}-\xi\right\|}{2^{k}}<\frac{\eta}{2} .
$$

Therefore, $\beta$ and $\gamma$ belong to $S(\alpha, \eta)$.

Due to the regularity the matrix $\mathscr{A}$ and Theorem A, it follows that

$$
\delta(\beta)=\lim _{n \rightarrow \infty} \sum_{k=1}^{\infty} a_{n k} \beta_{k}=\lim _{n \rightarrow \infty} \sum_{k=1}^{k_{1}} a_{n k} \alpha_{k}
$$

and

$$
\delta(\gamma)=\lim _{n \rightarrow \infty} \sum_{k=1}^{\infty} a_{n k} \gamma_{k}=\lim _{n \rightarrow \infty} \sum_{k=1}^{k_{1}} a_{n k} \alpha_{k}+\lim _{n \rightarrow \infty} \sum_{k>k_{1}} a_{n k} \xi .
$$

Since $\lim _{n \rightarrow \infty} \sum_{k>k_{1}} a_{n k}>\frac{1}{2}$, we have $\left\|\sum_{k>k_{1}} a_{n k} \xi\right\|=\left|\sum_{k>k_{1}} a_{n k}\right|\|\xi\|>\frac{1}{2}$ for every $n \in \mathbb{N}$ sufficiently large. Thus,

$$
\|\delta(\gamma)-\delta(\beta)\|=\left\|\lim _{n \rightarrow \infty} \sum_{k>k_{1}} a_{n k} \xi\right\|>\frac{1}{2} .
$$

Therefore the operator $\delta$ is discontinuous at each element $\alpha$ of $\Omega$. Since $\Omega$ is a complete metric space, the set of all discontinuity points of $\delta$ is the set of the second Baire category in $\Omega$ - a contradiction.

We introduce the notion of FK-space (see [1]).

Definition 1. A complete metric linear space $(B, d)$ of sequences, i. e., $B \subset b$ (see (1)), is said to be an FK-space provided that the linear operators $\delta_{k}: B \rightarrow X$,

$$
\delta_{k}(\alpha)=\alpha_{k}, \quad \alpha=\left(\alpha_{k}\right) \in B, \quad k=1,2 \ldots,
$$

are continuous on $B$.

Proposition 1. Let $\left(X,\|\cdot\|_{1}\right)$ be a Banach space. Let $(B, d)$ be a complete metric linear space of sequences of elements of $X$. Then $(B, d)$ is an FK-space if and only if the convergence in the sense of the metric $d$ implies the pointwise convergence in the sense of the norm $\|\cdot\|_{1}$ for each $k=1,2, \ldots$.

Proof. Let $(B, d)$ be an FK-space. The linear operator $\delta_{k}: B \rightarrow X, \delta_{k}(\alpha)=\alpha_{k}$, is continuous for each $k=1,2, \ldots$. According to the Heine definition of continuity, the operator $\delta_{k}: B \rightarrow X$ is continuous if for each sequence $\left(\alpha^{(r)}\right)_{r=1}^{\infty}, \alpha^{(r)}=\left(\alpha_{s}^{(r)}\right)_{r=1}^{\infty}$, of elements of $B$ such that $\alpha^{(r)} \rightarrow \alpha=\left(\alpha_{s}\right)$ as $r \rightarrow \infty$ by the metric $d$, we have $\delta_{k}\left(\alpha^{(r)}\right) \rightarrow \delta_{k}(\alpha)$ by the norm $\|\cdot\|_{1}$ as $r \rightarrow \infty$. In our case, $\delta_{k}\left(\alpha^{(r)}\right)=\alpha_{k}^{(r)}$ and $\delta_{k}(\alpha)=$ $\alpha_{k}$ for each $r=1,2, \ldots$. Consequently, $\alpha_{k}^{(r)} \rightarrow \alpha_{k}$ as $r \rightarrow \infty$. 
Now we denote by $F(\mathscr{A})=\left\{\alpha=\left(\alpha_{k}\right): \alpha_{k} \in B, k=1,2, \ldots: \lim _{n \rightarrow \infty} \sum_{k=1}^{\infty} a_{n k} \alpha_{k}\right.$ exists and equals $c$ for $\left.\alpha_{k} \rightarrow c\right\}$.

In the proof of Theorem 1 we shall use the following form of Banach's Subgroup Theorem (see [3]): If $G$ is any proper subgroup of a linear topological space E, then either $G$ is of the first category in $E$, or $G$ fails to satisfy the condition of Baire.

Theorem 1. Let $(B, d)$ be an FK-space, $B_{\infty} \subset B \subset b$. Let $\mathscr{A}=\left(a_{n k}\right)$ be a regular matrix of real numbers. Then the set $F(\mathscr{A})$ is of the first Baire category in $B$ for every regular matrix $\mathscr{A}$.

Proof. We set $\delta_{n s}(\alpha)=\sum_{k=1}^{s} a_{n k} \alpha_{k}, \alpha=\left(\alpha_{k}\right) \in B, n, s=1,2, \ldots$ From the above assumption it follows that each of the operators $\delta_{n s}: B \rightarrow X$ is continuous on $B$. Thus, $\delta_{n}(\alpha)=\lim _{s \rightarrow \infty} \delta_{n s}(\alpha)$ is a linear operator of the first Baire class defined on the set $D_{n}=\left\{\alpha \in B: \lim _{s \rightarrow \infty} \delta_{n s}(\alpha)\right.$ exists $\}$.

Obviously,

$$
\begin{aligned}
D_{n}=\{\alpha \in B: \forall p=1,2, \ldots & \left.\exists s_{0} \in \mathbb{N} \forall s, q \geq s_{0}:\left\|\delta_{n s}(\alpha)-\delta_{n q}(\alpha)\right\|_{1} \leq \frac{1}{p}\right\} \\
& =\bigcap_{p=1}^{\infty} \bigcup_{s_{0}=1}^{\infty} \bigcap_{s \geq s_{0}} \bigcap_{q \geq s_{0}}\left\{\alpha \in B:\left\|\delta_{n s}(\alpha)-\delta_{n q}(\alpha)\right\|_{1} \leq \frac{1}{p}\right\} .
\end{aligned}
$$

Let $s>q$. Then every operator $\left\|\delta_{n s}(\alpha)-\delta_{n q}(\alpha)\right\|_{1}$ is continuous, every set $D_{n}$ is an $F_{\sigma \delta}$ set. The common domain of all the operators $\delta_{n}$, the set $\bigcap_{n=1}^{\infty} D_{n}$, is also an $F_{\sigma \delta}$ set.

$$
\begin{aligned}
& \text { Put } S(\mathscr{A})=\bigcap_{n=1}^{\infty} D_{n} \text {. Then } F(\mathscr{A}) \subset S(\mathscr{A}) \text { and } \\
& \begin{aligned}
F(\mathscr{A})=\left\{\alpha \in S(\mathscr{A}): \lim _{n \rightarrow \infty} \delta_{n}(\alpha) \text { exists }\right\} \\
=\left\{\alpha \in S(\mathscr{A}): \forall p=1,2, \ldots \exists n_{0} \in \mathbb{N} \forall m, n \geq n_{0}:\left\|\delta_{n}(\alpha)-\delta_{m}(\alpha)\right\|_{1} \leq \frac{1}{p}\right\} \\
\quad=\bigcap_{p=1}^{\infty} \bigcup_{n_{0}=1}^{\infty} \bigcap_{n \geq n_{0}} \bigcap_{m \geq n_{0}}\left\{\alpha \in S(\mathscr{A}):\left\|\delta_{n}(\alpha)-\delta_{m}(\alpha)\right\|_{1} \leq \frac{1}{p}\right\} .
\end{aligned}
\end{aligned}
$$

Each of the operators $\left\|\delta_{n}(\alpha)-\delta_{m}(\alpha)\right\|_{1}$ is a Baire one operator, hence the set $F(\mathscr{A})$ is a $G_{\delta \sigma \delta}$ set, therefore $F(\mathscr{A})$ satisfies the condition of Baire. Since $F(\mathscr{A})$ is a proper subgroup of $B$, Theorem 1 is a consequence of Banach's Subgroup Theorem.

The following Lemma is proved in [5].

Lemma 2. Let $Z$ be a convex nowhere dense set in a Banach space $X$. Then $Z$ is strongly porous in $X$.

We shall show that in the set $B_{\infty}$ endowed with the norm $\|\alpha\|_{2}=\sup \left\{\left\|\alpha_{k}\right\|_{1}\right\}$, $\alpha=\left(\alpha_{k}\right) \in B_{\infty}$, the set $F(\mathscr{A})$ is strongly porous. 
Theorem 2. In the Banach space $\left(B_{\infty},\|\cdot\|_{2}\right)$ the set $F(\mathscr{A})$ is strongly porous in $B_{\infty}$ for any regular matrix $\mathscr{A}$.

Proof. Each of the operators $\delta_{n}: B_{\infty} \rightarrow X, \delta_{n}(\alpha)=\sum_{k=1}^{\infty} a_{n k} \alpha_{k}, n=1,2, \ldots$, fulfils the Lipschitz condition with the same constant $M$.

Due to Theorem A, for $\alpha=\left(\alpha_{k}\right) \in B_{\infty}, \beta=\left(\beta_{k}\right) \in B_{\infty}$, we have

$$
\left\|\delta_{n}(\alpha)-\delta_{n}(\beta)\right\|_{1}=\left\|\sum_{k=1}^{\infty} a_{n k}\left(\alpha_{k}-\beta_{k}\right)\right\|_{1} \leq\|\alpha-\beta\|_{2} \sum_{k=1}^{\infty}\left|a_{n k}\right| \leq M\|\alpha-\beta\|_{2} .
$$

The set $F(\mathscr{A})$ is a subspace of $B_{\infty}$ and it is convex. We show that $B_{\infty} \backslash F(\mathscr{A})$ is open in $B_{\infty}$. Let $\alpha=\left(\alpha_{k}\right) \in B_{\infty} \backslash F(\mathscr{A})$. Then the sequence $\left(\delta_{n}(\alpha)\right)$ does not satisfy Cauchy's conditions, i. e.,

$$
\exists \varepsilon>0 \forall n_{0} \in \mathbb{N} \exists m, n>n_{0}:\left\|\delta_{n}(\alpha)-\delta_{m}(\alpha)\right\|_{1} \geq \varepsilon .
$$

Let $\beta=\left(\beta_{k}\right) \in B_{\infty}$ such that

Then, by (5), we have

$$
\|\alpha-\beta\|_{2}<\frac{\varepsilon}{4 M}
$$

$$
\begin{aligned}
\varepsilon \leq \| \delta_{n}(\alpha)- & \delta_{m}(\alpha) \|_{1} \\
& \leq\left\|\delta_{n}(\alpha)-\delta_{n}(\beta)\right\|_{1}+\left\|\delta_{n}(\beta)-\delta_{m}(\beta)\right\|_{1}+\left\|\delta_{m}(\beta)-\delta_{m}(\alpha)\right\|_{1} \\
< & 2 M\|\alpha-\beta\|_{2}+\left\|\delta_{n}(\beta)-\delta_{m}(\beta)\right\|_{1} .
\end{aligned}
$$

By (6), we have

$$
\frac{\varepsilon}{2}<\left\|\delta_{n}(\beta)-\delta_{m}(\beta)\right\|_{1}
$$

Thus, $\beta=\left(\beta_{k}\right) \notin F(\mathscr{A})$ and

$$
S\left(\alpha, \frac{\varepsilon}{4 M}\right) \subset B_{\infty} \backslash F(\mathscr{A}),
$$

which means that $B_{\infty} \backslash F(\mathscr{A})$ is open in $B_{\infty}$.

Therefore, $F(\mathscr{A})$ is a closed nowhere dense subset of $B_{\infty}$, and Theorem 2 follows from Lemma 2.

\section{REFERENCES}

[1] Bennet, G. and Kalton, N. J.: Consistency theorems for almost convergence, Trans. Amer. Math. Soc., 198 (1974), 23-43.

[2] Connor, J.: A short proof of Steinhaus' theorem on summability, Amer. Math. Monthly, 92 (1985), $420-421$.

[3] Kelley, J. L. and Namioka, I.: Linear Topological Spaces, New York, 1963.

[4] Kostyrko, P.: Convergence fields of regular matrix transformations, Tatra Mount. Math. Publ., 28 (2004), 153-157.

[5] OlevskiI, V.: A note on the Banach-Steinhaus theorem, Real Anal. Exchange, 17 (1991-1992), 399-401.

[6] Petersen, G.M.: Regular matrix transformations, London, 1966. 
[7] ŠALÁT, T.: On convergence fields of regular matrix transformations, Czechoslovak Math. J., 26 (1976), No. 101, 613-627.

[8] SiкоRsкi, R.: Funkcje rzeczywiste. I, Warszawa, 1958.

[9] VISNYAI, T.: Limitation Methods of Sequences of Elements of a Banach Space [in Slovak], Author's Abstract of PhD Thesis, Bratislava, 2002.

[10] Zajíček, L.: Porosity and $\sigma$-porosity, Real Anal. Exchange, 13 (1987-1988), 314-350.

\section{Author's address}

\section{T. Visnyai:}

Comenius University, Faculty of Mathematics, Physics and Informatics, Mlynská dolina, 842 48, Bratislava 4, SLovakia

E-mail address: visnyai@zoznam.sk 